\title{
Efecto de la incentivación de las abejas con carbohidratos sobre la composición isotópica de ${ }^{13} \mathrm{C}$ de mieles de Uruguay
}

\section{Effect of energetic incentive of bees with carbohydrates on the ${ }^{13} \mathrm{C}$ isotopic composition of honeys from Uruguay}

\author{
Berriel, Verónica (1) \\ (1) Centro de Aplicaciones de Tecnología Nuclear en Agricultura Sostenible, Departamento de Suelos y Aguas, \\ Facultad de Agronomía, Universidad de la República, Montevideo, Uruguay. \\ Contacto: vberriel@fagro.edu.uy
}

RECIBIDO: 30/5/2018 - APROBADO: 9/10/2018

\begin{abstract}
Resumen
En el mercado internacional, las mieles son sometidas a una rigurosa prueba analítica que determina su autenticidad o su adulteración con azúcares artificiales derivados de plantas C-4. En caso de mal manejo de la incentivación, es posible que la prueba de autenticidad dé como resultado que las mieles están adulteradas con azúcares exógenos. El objetivo de este trabajo fue evaluar la incidencia de la práctica de incentivación local en la prueba analítica de determinación de adulteración por azúcares C-4. Para este estudio fueron colectadas mieles de 20 apiarios. Los resultados indicaron que el manejo de los apiarios según las normas de buenas prácticas de los apicultores no revisten peligro de que las mieles sean catalogadas como adulteradas. Sin embargo, se encontró que en un apiario donde se incentivó a las abejas en un período no oportuno la prueba de autenticidad dio como resultado que la miel estaba adulterada por azúcares C-4. Por lo tanto, es recomendable que la práctica de incentivación sea realizada solo en momentos de escasez de alimentos para las abejas, para que estas no acopien el mismo alimento y los controles analíticos demuestren el alto estándar de calidad de las mieles uruguayas.

Palabras clave: miel, adulteración con azúcares $\mathrm{C}-4,{ }^{13} \mathrm{C}$.

Abstract

In the international market, honeys are subjected to a rigorous analytical test that determines their authenticity or adulteration with artificial sugars derived from C-4 plants. In case of mishandling of the supply of the energetic incentive, it is possible that the honeys result in this authenticity test as adulterated with exogenous sugars. The objective of this work was to evaluate the incidence of the practice of feeding whit energetic incentive in the analytical test of determination of adulteration by C-4 sugars. For this study honey from 20 apiaries were collected. The results showed that the apiary-handling carried out according to the standards of good practices of beekeepers are not risk for honey to be classified as adulterated. However, it was found that in an apiary where bees had an energetic incentive in a not opportune period, the honey resulted in the authenticity proof as adulterated by C-4 sugars. Therefore, it was recommended that the practice of energetic incentive be carried out only in times of food shortage for the bees, so that they do not collect the same food, and the analytical controls demonstrate the high quality standard of Uruguayan honeys.

Keywords: honey, C-4 sugar adulteration, ${ }^{13} \mathrm{C}$.
\end{abstract}

\section{Introducción}

La miel se define como una sustancia dulce natural producida por abejas Apis mellifera a partir del néctar o de las secreciones de las plantas, o de las excreciones de insectos succionadores de plantas. Durante la síntesis de la miel ocurre la combinación de las sustancias extraídas de las plantas con las propias secreciones, luego esta mezcla se deposita, deshidrata y por último añeja en el panal (Bogdanov y Martin, 2002).

De la definición anterior se desprende que cualquier sustancia exógena agregada a la miel de manera voluntaria (por ejemplo, para incrementar el volumen del producto) o involuntaria (por ejemplo, durante la alimentación de las abejas) es considerada una adulteración (Guler, et al., 2014; Borovšak, et al., 2017). Sin embargo, el bajo costo de los jarabes y la dificultad de su detección han hecho de este método de adulteración el más frecuente (Naila, et al., 2018).

Una práctica relativamente expandida entre los apicultores de Uruguay es la incentivación de las abejas. La incentivación consiste en suministrar carbohidratos a las abejas cerca del final del invierno para que estén en óptimas condiciones al comienzo de la floración (Bazzurro, 2000). Si esta práctica 
no se lleva a cabo de manera controlada, presupone un riesgo inminente de contaminación de la miel con azúcares C-4 (Bazzurro, 2000) y que, por lo tanto, pueda ser catalogada como miel adulterada por azúcares exógenos.

Para la detección de azúcares C-4 en la miel se realiza un análisis de la composición isotópica de ${ }^{13} \mathrm{C}$ o valor $\delta^{13} \mathrm{C}$ en muestras de miel (White y Winters, 1989). Para determinar si una miel fue adulterada es necesario tener en cuenta que los valores $\delta^{13} \mathrm{C}$ de las mieles son un reflejo del valor isotópico $\delta^{13} \mathrm{C}$ de las plantas de las cuales se originó. Se puede afirmar entonces que en general las mieles provienen de plantas con ciclo fotosintético C-3, mientras que los adulterantes derivan de plantas con ciclo fotosintético C-4 (White y Winters, 1989). Debido a las diferencias bioquímicas entre plantas con distintos ciclos fotosintéticos, los valores $\delta^{13} \mathrm{C}$ se ubican en distintos rangos numéricos. Los valores $\delta^{13} \mathrm{C}$ de las plantas C-4 (adulterantes) son significativamente mayores que los valores $\delta{ }^{13} \mathrm{C}$ de plantas C-3 (De Niro y Epstein, 1978).

El método oficial actual se basa en la utilización del valor de $\delta^{13} \mathrm{C}$ de la proteína de la miel como un estándar interno (White y Winters, 1989). Este criterio se fundamenta en que en una muestra genuina los valores de $\delta^{13} \mathrm{C}$ de la miel y de su proteína deben ser muy similares, ya que el agregado de azúcares solo altera el valor de $\delta^{13} \mathrm{C}$ de la miel pero no el de sus proteínas (White y Winters, 1989; Padovan, et al., 2007).

El criterio internacional actual establece que una miel está adulterada cuando la diferencia entre el valor de $\delta^{13} \mathrm{C}$ de las proteínas extraídas y la miel (índice isotópico) es inferior a -1,0 $\%$ (Berriel y Perdomo, 2016). El método del estándar interno permite además calcular la proporción de adulterante C-4 agregado, ya que existe una relación directa entre la diferencia isotópica proteína-miel y la proporción de adulterante agregado $(-1,0 \%$ equivale a $7 \%$ de azúcar C- 4 agregado, asumiendo un valor de composición isotópica de ${ }^{13} \mathrm{C}$ del jarabe de -9,7\%o).

El objetivo de este estudio es determinar si las prácticas locales de incentivación de las abejas impactan en estos valores isotópicos de ${ }^{13} \mathrm{C}$ de las mieles.

\section{Materiales y Métodos}

De una colección de muestras de mieles de pradera de Uruguay, cosechadas entre los años 2008 y 2012, se eligieron 20 al azar. Los apiarios de los cuales provenían las mieles estaban distribuidos a lo largo del país. Los apicultores, inscriptos ante el Ministerio de Ganadería, Agricultura y Pesca de Uruguay (MGAP), declararon el origen genuino de sus muestras. De ellos, seis declararon que no alimentaron a las abejas en ningún momento del año, 13 indicaron que incentivaron a las abejas con azúcares C- 4 antes del inicio de la floración, y un apicultor declaró haber incentivado luego de iniciado el flujo de néctar, próximo al período de la cosecha de la miel. La incentivación consistió en mezclar partes iguales de azúcar de caña y agua (masa: volumen) o partes iguales de jarabe de alta fructosa y agua (volumen: volumen).

Las muestras de miel fueron filtradas por un tamiz de 0,10 $0,15 \mathrm{~mm}$. Las proteínas fueron aisladas de acuerdo al método del estándar interno AOAC 991.41 (AOAC International, 1995). Las muestras fueron extraídas de la siguiente forma: $10 \mathrm{~g}$ de miel se disolvieron en $4 \mathrm{~mL}$ de agua destilada. Luego, $2 \mathrm{~mL}$ de tungstato de sodio al $10 \%(\mathrm{~m} / \mathrm{v})$ y $2 \mathrm{~mL}$ de ácido sulfúrico $0,67 \mathrm{~N}$ se mezclaron y agregaron a la solución de miel. La mezcla de reacción se homogeneizó y calentó a $80^{\circ} \mathrm{C}$ hasta la completa floculación de las proteínas; en el caso de no ocurrir floculación, se agregaron alícuotas de $2 \mathrm{~mL}$ de ácido sulfúrico $0,67 \mathrm{~N}$ hasta la observación de floculación. La mezcla fue centrifugada por 5 minutos a 1500xg. Luego se eliminó el sobrenadante, y el pellet fue lavado y centrifugado tres veces con $50 \mathrm{~mL}$ de agua destilada. Las proteínas purificadas fueron secadas durante 3 horas en estufa a $75^{\circ} \mathrm{C}$.

Tanto las muestras de miel como de proteína fueron posteriormente analizadas por triplicado mediante EA-IRMS, de acuerdo al método oficial AOAC 978.17 (AOAC International, 1995). Las muestras de miel y proteína se colocaron en cápsulas de estaño antes de ser introducidas en un analizador elemental (Flash EA, 1112 series) acoplado a un espectrómetro de masas de relaciones isotópicas (Thermo Finnigan Delta Plus) mediante la interfase CONFLO III. Los valores de $\delta^{13} \mathrm{C}$ se estimaron de:

$$
\delta(\%)=\frac{(\mathrm{R} \text { muestra }-\mathrm{R} \text { estándar }) \times 1000}{\mathrm{R} \text { estándar }}
$$

donde $\mathrm{R}$ es la relación entre los valores de ${ }^{45} \mathrm{CO}_{2} /{ }^{46} \mathrm{CO}_{2}$ para la muestra y el estándar (Viena Pee Dee Belemnite).

Para la normalización de los datos isotópicos se utilizaron los estándares IAEA CH-6 y USGS-40, con valores $\delta^{13} \mathrm{C}$ certificados de $-10,449 \%$ y $-26,39 \%$, respectivamente.

La proporción aparente de azúcares C-4 fue calculada de acuerdo a:

$$
\text { Azúcares C }-4(\%)=\frac{\delta^{13} C_{P}-\delta{ }^{13} C_{M}}{\delta{ }^{13} C_{P}-(-9,7)} \times 100
$$

donde $\delta^{13} C_{p}$ y $\delta^{13} C_{M}$ son los valores de $\delta^{13} \mathrm{C}(\%)$ de la proteína y la miel, respectivamente, y $-9,7$ es el valor estándar internacional de $\delta^{13} \mathrm{C}(\% 0)$ del jarabe de alta fructosa, tomado del valor promedio encontrado por White y Winters (1989).

Para controlar el buen desempeño del método a una muestra genuina, producida dentro de un predio experimental de la Facultad de Agronomía, se le agregó cantidades crecientes de jarabe de maíz de alta fructosa entre 5 y $82 \%(\mathrm{~m} / \mathrm{m})$. Sobre las mezclas miel-jarabe se determinaron los valores $\delta^{13} \mathrm{C}$ y las proteínas extraídas. La función que correlacionó el $\%(\mathrm{~m} / \mathrm{m})$ de jarabe determinado mediante el método de análisis $(\mathrm{y})$ con el \% $(\mathrm{m} / \mathrm{m})$ de la mezcla preparada $(\mathrm{x})$ fue: $\mathrm{y}=1,0024 \mathrm{x}+0,7509 \mathrm{y}$ presentó un $\mathrm{R}^{2}=0,9991$.

Los análisis estadísticos se realizaron con las rutinas incluidas en el programa XLStat, Addinsoft, 2018.

\section{Resultados y Discusión}

\section{Valores $\delta^{13} \mathrm{C}$ de composición isotópica}

Los valores $\delta^{13} \mathrm{C}$ de las mieles oscilaron entre $-26,23$ y $-22,75 \%$, mientras que los valores de las proteínas variaron entre $-25,95$ y $-24,85 \%$ (Tabla 1 ). Del total de las muestras, 19 presentaron índices isotópicos de entre $-0,24$ y $0 \%$, por lo tanto, fueron clasificadas como mieles genuinas sin agregado de azúcares C-4, de acuerdo a los estándares internacionales.

Una excepción en el conjunto de muestras fue la \#20, cuyo índice isotópico $(-2,1 \%)$ excedió el límite de corte (-1,0\%o). Por tal razón esta muestra fue catalogada como no genuina 
o adulterada por agregado de azúcares C-4. La información asociada a esta muestra indicaba que las abejas habían sido incentivadas en un período próximo a la cosecha. En función de los valores isotópicos obtenidos se evidenció el impacto negativo de la práctica de incentivación luego del inicio de la floración sobre la calidad de la miel.

Para los análisis estadísticos siguientes, la muestra \#20 se eliminó del conjunto de datos por tratarse claramente de un resultado adverso.

Para este conjunto de las mieles genuinas los valores máximos $\delta^{13} \mathrm{C}$ de las mieles y sus proteínas extraídas fueron $-25,08$ y $-25,17 \%$, respectivamente (Tabla 1 ).

El rango de variación de los valores isotópicos en las mieles genuinas fue un indicador de que todas ellas tuvieron un origen botánico C-3 (De Niro y Epstein, 1978; White y Winters, 1989).
Además, la distribución de estos valores de composición isotópica tanto de las mieles como los de las proteínas extraídas fueron normales de acuerdo al test Shapiro-Wilk ( $p>0,5)$.

Cabe resaltar que entre los tratamientos con y sin incentivación los valores isotópicos de $\delta^{13} \mathrm{C}$ de la miel y $\delta^{13} \mathrm{C}$ de las proteínas extraídas no presentaron diferencias significativas ( $>0,05)$. Por lo tanto, resulta evidente que la práctica de incentivación de las abejas realizada de manera adecuada no tiene incidencia en los valores de composición isotópica de ${ }^{13} \mathrm{C}$ de las mieles.

Las incertidumbres de los valores $\delta^{13} \mathrm{C}$ de la miel y sus proteínas (Tabla 1) se distribuyeron de manera similar entre ambos tratamientos y en concordancia con estudios previos de estimaciones de la incertidumbre reportados por Berriel y Constantino (inédito).

\begin{tabular}{|c|c|c|c|c|c|c|}
\hline \multirow[t]{2}{*}{ Muestra } & \multirow[t]{2}{*}{ Tratamiento $^{1}$} & $\delta^{13} \mathrm{C}_{\mathrm{P}} \pm \mathrm{U}_{\delta}^{13} \mathrm{Cp}^{2}$ & $\delta^{13} \mathrm{C}_{\mathrm{M}} \pm \mathrm{U}_{\delta}{ }^{13} \mathrm{Cm}^{3}$ & \multirow[t]{2}{*}{$\begin{array}{c}\text { Clasificación } \\
\text { de la miel }\end{array}$} & $\begin{array}{c}\text { Porcentaje de } \\
\text { adulteración } \\
\text { calculado }\end{array}$ & $\begin{array}{c}\text { Porcentaje de } \\
\text { adulteración } \\
\text { informado } \pm \mathrm{U}_{\% \mathrm{C} 4}{ }^{5}\end{array}$ \\
\hline & & \multicolumn{2}{|c|}{$\%$} & & \multicolumn{2}{|r|}{$\%$} \\
\hline 1 & Sin & $-25,66 \pm 0,15$ & $-25,76 \pm 0,18$ & $\mathrm{P}$ & 0 & $\mathrm{ND}^{6}$ \\
\hline 2 & Sin & $-25,70 \pm 0,12$ & $-25,82 \pm 0,17$ & $\mathrm{P}$ & 0 & $\mathrm{ND}$ \\
\hline 3 & Sin & $-25,65 \pm 0,16$ & $-25,70 \pm 0,15$ & $\mathrm{P}$ & 0 & ND \\
\hline 4 & Sin & $-25,71 \pm 0,13$ & $-26,10 \pm 0,11$ & $\mathrm{P}$ & 0 & $\mathrm{ND}$ \\
\hline 5 & Sin & $-25,71 \pm 0,13$ & $-25,69 \pm 0,16$ & $\mathrm{P}$ & 0,11 & $\mathrm{ND}$ \\
\hline 6 & Sin & $-25,54 \pm 0,16$ & $-25,51 \pm 0,16$ & $\mathrm{P}$ & 0,18 & ND \\
\hline 7 & Con & $-25,87 \pm 0,16$ & $-25,88 \pm 0,14$ & $\mathrm{P}$ & 0 & ND \\
\hline 8 & Con & $-25,63 \pm 0,16$ & $-25,76 \pm 0,19$ & $\mathrm{P}$ & 0 & ND \\
\hline 9 & Con & $-25,28 \pm 0,11$ & $-25,52 \pm 0,15$ & $\mathrm{P}$ & 0 & ND \\
\hline 10 & Con & $-25,32 \pm 0,14$ & $-25,62 \pm 0,12$ & $\mathrm{P}$ & 0 & $\mathrm{ND}$ \\
\hline 11 & Con & $-25,73 \pm 0,16$ & $-26,16 \pm 0,19$ & $\mathrm{P}$ & 0 & ND \\
\hline 12 & Con & $-25,76 \pm 0,12$ & $-26,23 \pm 0,11$ & $\mathrm{P}$ & 0 & ND \\
\hline 13 & Con & $-25,60 \pm 0,14$ & $-25,60 \pm 0,18$ & $\mathrm{P}$ & 0,01 & ND \\
\hline 14 & Con & $-25,95 \pm 0,15$ & $-25,93 \pm 0,16$ & $\mathrm{P}$ & 0,14 & ND \\
\hline 15 & Con & $-25,44 \pm 0,13$ & $-25,39 \pm 0,15$ & $\mathrm{P}$ & 0,32 & $\mathrm{ND}$ \\
\hline 16 & Con & $-25,36 \pm 0,16$ & $-25,31 \pm 0,14$ & $\mathrm{P}$ & 0,37 & $\mathrm{ND}$ \\
\hline 17 & Con & $-25,17 \pm 0,15$ & $-25,08 \pm 0,10$ & $\mathrm{P}$ & 0,53 & ND \\
\hline 18 & Con & $-25,76 \pm 0,15$ & $-25,55 \pm 0,11$ & $\mathrm{P}$ & 1,29 & ND \\
\hline 19 & Con & $-25,87 \pm 0,12$ & $-25,63 \pm 0,16$ & $\mathrm{P}$ & 1,46 & $\mathrm{ND}$ \\
\hline 20 & Con & $-24,85 \pm 0,17$ & $-22,75 \pm 0,15$ & A & 13,87 & $13,9 \pm 1,1$ \\
\hline
\end{tabular}

Tabla 1. Valores de $\delta^{13} \mathrm{C}$ de miel y sus proteínas de diferentes apiarios de Uruguay, clasificación de la miel en pura (P) o adulterada (A) según valor de rechazo internacional, y proporción estimada de adulterante C4 agregado a la miel.

TTratamiento: $\operatorname{Sin}=$ sin incentivación de la colmena, $\mathrm{Con}=$ con incentivación de la colmena.

${ }^{2} \mathrm{U}_{\delta}{ }^{13} \mathrm{Cp}$ : incertidumbre expandida estimada para el valor $\delta^{13} \mathrm{C}$ de la proteína extraída de la miel.

${ }^{3} \mathrm{U}_{\delta}{ }^{13} \mathrm{Cm}$ : incertidumbre expandida estimada para el valor $\delta^{13} \mathrm{C}$ de la miel.

${ }^{4}$ Clasificación de la miel: P: miel pura o auténtica; A: miel adulterada con azúcares C-4.

${ }^{5} \mathrm{U}_{\% c 4}$ : incertidumbre expandida estimada para el porcentaje de adulteración con azúcares C-4.

${ }^{6} \mathrm{ND}=$ no detectado. 


\section{Estimación del porcentaje de azúcares C-4 en miel}

Los porcentajes estimados de adulteración de las mieles con azúcares C-4 variaron entre 0 y $1,5 \%(\mathrm{~m} / \mathrm{m})$. A pesar de que la media en este parámetro fue superior para el grupo de mieles que provenían de apiarios que habían recibido incentivación $(0,31 \%)$, no fue significativamente distinta de la media del grupo sin incentivar (0,09\%), de acuerdo al análisis ANOVA y Test de LSD-Fischer ( $\mathrm{p}>0,05)$. También es importante mencionar que los valores de porcentaje de adulteración obtenidos mediante el cálculo de la Ecuación 2 están por debajo del límite de detección del método, por lo tanto, el resultado a informar fue "no detectado".

Además, el porcentaje de adulteración estimado para la miel \#20 fue $13,9 \%(\mathrm{~m} / \mathrm{m})$, que indicaría que por cada $100 \mathrm{~g}$ de miel casi $14 \mathrm{~g}$ correspondían a azúcares exógenos derivados de plantas C-4 (como la caña de azúcar o el maíz). La normativa internacional indica que toda miel con porcentajes de adulteración de azúcares C-4 superiores al 7\% $(\mathrm{m} / \mathrm{m})$ sea considerada no genuina. En este caso, probablemente debido a que los carbohidratos fueron suministrados luego del inicio del flujo de néctar en el ambiente, estos fueron acumulados en los panales al no ser consumidos en su totalidad por las abejas (Bazzurro, 2000). A la luz de este resultado es interesante desarrollar ensayos experimentales que estudien la dinámica de consumo de los azúcares C-4 por las abejas y conocer la variabilidad de la composición isotópica de carbono de dichas mieles. El factor momento de la incentivación en esos ensayos resulta ser clave, por lo tanto deberían considerarse al menos dos épocas de incentivación: una fuera de períodos de mielada y otra en el periodo de mielada.

\section{Conclusiones}

Los resultados obtenidos revelaron que en líneas generales la práctica de incentivación llevada a cabo en Uruguay no repercute negativamente en los valores de composición isotópica de las mieles, ni afecta su condición de alimento genuino. Sin embargo, de acuerdo a los estándares internacionales una muestra se clasificó como adulterada con azúcares C-4. Probablemente este resultado adverso se debió a que la incentivación de las abejas fue llevada a cabo luego de que comenzaran los flujos de néctar.

Dada la importancia de la apicultura a nivel productivo en Uruguay, es importante que las prácticas de incentivación sean realizadas fuera de los periodos de fuerte flujo de néctar hacia la colmena, de manera de alcanzar los estándares de calidad exigidos en el mercado internacional.

\section{Reconocimientos}

Agradezco a los apicultores que gentilmente proporcionaron muestras de miel e información de manejo asociado a la práctica apícola para este estudio. También destaco la colaboración del Ing. Agr. Pablo Cracco (FAgro) por sus interesantes aportes a la interpretación de la información de campo. Agradecezco muy especialmente a Pablo Constantino (LATU) por el desarrollo de los modelos y planillas de cálculo para estimar la incertidumbre de las composiciones isotópicas de carbono y del porcentaje de adulteración. Por ultimo, también quiero destacar el aporte realizado por revisores anónimos quienes mediante sus comentarios constructivos permitieron mejorar el manuscrito original.

\section{Referencias}

AOAC International, 1995. Official Methods of Analysis of AOAC International. 16a ed. Gaithersburg: AOAC. Official Method 978.17, first action 1978-final action 1979.

Bazzurro, D., 2000. Manejo del apiario para incrementar la cosecha de miel y obtener un producto de alta calidad [En línea]. [s.l.]: [s.n.]. [Consulta 24 de abril de 2018]. Disponible en: http://www.abejasprepirineo.com/archivos/ ManejoApiario.pdf

Berriel, C. y Constantino, P. Uncertainty estimation of the percentage of high-fructose corn syrup added tohoney by EA-IRMS. Inédito.

Berriel, V. y Perdomo, C., 2016. Determination of high fructose corn syrup concentration in Uruguayan honey by 13C analyses. En: LWT - Food Science and Technology, 73, pp.649-653.

Bogdanov, S. y Martin P., 2002. Honey authenticity: a review, Mitteilungen En: Aus Dem Gebiete Der Lebensmittel, 93, pp.232-254.

Borovšak, K., Ogrinc, N., Lilek, N. y Korošec, M., 2017. Feeding honey-bee colonies (Apis mellifera carnica Poll.) and detection of honey adulteration A. En: Acta Alimentaria, 46(2), pp.127-136.

De Niro, M.J. y Epstein, S., 1978. Influence of diet on the distribution of carbon isotopes. En: Geochimica and Cosmochimica Acta, 12, pp.133-149.

Guler, A., Kocaokutgen, H., Garipoglu A. V., Onder, H., Ekinci, D. y Biyik, S., 2014. Detection of adulterated honey produced by honeybee (Apis mellifera L.) colonies fed with different levels of commercial industrial sugar (C3 and $\mathrm{C} 4$ plants) syrups by the carbon isotope ratio analysis. En: Food Chemistry, 155, pp.155-160.

Naila, A., Flint, S. H., Sulaiman, A.Z. y Zuben Weeds, A-A., 2018. Classical and novel approaches to the analysis of honey and detection of adulterants. En: Food Control, 90, pp.152-165.

Padovan, G. J., Rodrigues, L. P., Leme, I. A., De Jong, D. y Marchini, J. S., 2007. Presence of C-4 sugars in honey samples detected by the carbon isotope ratio measured by IRMS. En: Eurasian Journal of Analytical Chemistry, 2(3), pp.134-141.

White, J.W. y Doner, L.W., 1978. The 13C/ 12C ratio in honey. En: Journal of Apicultural Research, 17, pp.94-99.

White, J.W. y Robinson, F.A., 1983. 13C/12C ratio of citrus honeys and their regulatory implications ratio. En: Journal of the Association of Official Analytical Chemists, 66, pp.1-3.

White, J.W. y Winters, K., 1989. Honey protein as internal standard for stable isotope ratio detection of adulteration of honey. En: Journal of the Association of Official Analytical Chemists, 72, pp.907-911. 